\title{
The emergence of Klebsiella pneumoniae liver abscess in non-diabetic patients and the distribution of capsular types
}

\author{
Chien Chuang ${ }^{1}$, Wen-Chien Fan ${ }^{2,3}$, Yi-Tsung Lin ${ }^{2,4^{*}}$ and Fu-Der Wang ${ }^{2,3}$
}

\begin{abstract}
Background: Klebsiella pneumoniae is the most common pathogen of community-acquired pyogenic liver abscess in East Asia. Diabetes mellitus (DM) is a well-established risk factor for K. pneumoniae liver abscess (KPLA). However, reports regarding the emergence of KPLA in non-diabetic patients are limited.

Results: A total 230 patients with KPLA from a medical center in Taiwan were identified retrospectively. The rate of DM in patients with KPLA was 44.4 \% in 2011, $57.9 \%$ in 2012, $44.9 \%$ in 2013, 35.0 \% in 2014, and $53.5 \%$ in 2015. Diabetic patients had higher rate of gas-forming abscesses than non-diabetic patients, but the clinical outcomes were not different. The six virulent capsular types (K1, K2, K5, K20, K54, and K57) accounted for $90.2 \%$ of all K. pneumoniae isolates, and were more prevalent in non-diabetic than diabetic patients ( $93.9 \mathrm{vs} 85.9 \%, P=0.048$ ). The six virulent capsular types were also more prevalent in the group with optimal glycemic levels (Non-DM and DM with HbA1c level $<7 \%$ ) than the DM group with $\mathrm{HbA1c}$ level $\geq 7 \%$ (93.9 vs $84.3 \%, P=0.022$ ).

Conclusion: Klebsiella pneumoniae liver abscess has emerged in non-diabetic patients in Taiwan. Diabetic patients were at higher risk of acquiring gas-forming abscesses. Non diabetic patients and diabetic patients with optimal glycemic levels are more susceptible to the virulent capsular types of K. pneumoniae.
\end{abstract}

Keywords: Capsular type, Diabetes, Hemoglobin A1c, Klebsiella pneumoniae, Liver abscess

\section{Background}

Klebsiella pneumoniae is known as a major pathogen to cause nosocomial urinary-tract infection, pneumonia, and intra-abdominal infections worldwide [1-3], as well as community-acquired pyogenic infection in Taiwan [4-6]. In the past three decades in East Asian countries (especially Taiwan, Korea, and Singapore), $K$. pneumoniae has emerged as the major cause of community-acquired pyogenic liver abscess [7-15]. A distinct invasive syndrome of Klebsiella pneumoniae liver abscess (KPLA) with extrahepatic complications involving endophthalmitis, infection in the central nervous system as well as septic metastatic lesions in other organs, have

\footnotetext{
*Correspondence: ytlin8@vghtpe.gov.tw

${ }^{2}$ Division of Infectious Diseases, Department of Medicine, Taipei Veterans General Hospital, Number 201, Section 2, Shih-Pai Road, Beitou District, Taipei 11217, Taiwan

Full list of author information is available at the end of the article
}

also been reported [16-18]. The prevalent KPLA is now considered as an endemic disease in Taiwan $[14,19]$. The mortality rate due to KPLA has decreased in Taiwan in recent years $[14,16]$. However, metastatic complications with ocular or neurological involvement from KPLA could result in catastrophic disability and a poor longterm prognosis [18]. The high economic burden resulting from KPLA is also the concern [9].

Diabetes mellitus (DM) is a well-established risk factor for KPLA. The percentage of DM in KPLA patients was as high as $60-78.4 \%$ in previous studies in Taiwan $[12,16,17,20]$. Patients with underlying DM are also more susceptible to septic metastatic complications from KPLA [12, 17, 20, 21]. Recently, we reported that diabetic patients with uncontrolled glycemia were more susceptible to metastatic complications from KPLA than those with controlled glycemia [11]. In contrast to the previous studies, we found that about half of the patients did not 
have DM from January 2007 to January 2012 [11]. However, the emergence of KPLA in non-diabetic patients has received little attention in the literature. The relationship between the capsular types of $K$. pneumoniae and different glycemic status of patients with KPLA remains undetermined.

In this study, we aimed to investigate the clinical characteristics of KPLA patients and the associated capsular types of $K$. pneumoniae strains in Taiwan in the recent 5 years. Particular attention was focused on the emergence of KPLA in non-diabetic patients.

\section{Methods}

\section{Study population and data collection}

This retrospective analysis enrolled the patients diagnosed with KPLA who were admitted to Taipei Veterans General Hospital (a 2900-bed tertiary medical center) from January 2011 to December 2015. We excluded patients aged $<20$ years, patients with polymicrobial or nosocomial infections, or patients who developed liver abscess after invasive procedure, including trans-hepatic arterial chemoembolization or stent replacement over common bile duct. The medical records of patients with KPLA were reviewed by two infection specialists. Information regarding clinical features, underlying diseases, laboratory findings, origin and nature of the liver abscesses, imaging findings, treatment and outcomes were collected. Assessment of blood glycemic status was based on hemoglobin A1c (HbA1c) levels at the time of infection or as close to the time of infection as possible, within 1 month before this episode of KPLA. Since newly diagnosed DM is not unusual in patients with KPLA, it is a common practice to check HbA1c in patients with KPLA in Taiwan. This study was approved by Institutional Review Board of Taipei Veterans General Hospital.

\section{Definition}

An episode of KPLA was defined as a culture-confirmed K. pneumoniae isolated from an abscess or blood and $\geq 1$ liver abscess, detected by sonography or computed tomography. Only the first episode of KPLA in an individual patient diagnosed at our hospital during the study period was included. The diagnosis of DM was according to the criteria published by the American Diabetes Association in 2012: HbA1c level $\geq 6.5 \%$, symptoms of hyperglycemia with a random plasma glucose $\geq 200 \mathrm{mg} / \mathrm{dL}$, or 2-h plasma glucose $\geq 200 \mathrm{mg} / \mathrm{dL}$ during an oral glucose tolerance test, or fasting plasma glucose $\geq 126 \mathrm{mg} / \mathrm{dL}$ [23]. "Cryptogenic KPLA" was that in which no obvious extra-hepatic source of infection could be identified. "Biliary-tract origin of a liver abscess" was defined if the clinical features of cholecystitis/cholangitis or extra-hepatic biliary ductal abnormalities were identified upon radiography [11]. "Chronic lung disease" included chronic obstructive pulmonary disease, bronchiectasis, or any structural lung disease with the exception of bronchogenic carcinoma. Chronic kidney disease was defined as baseline serum creatinine $\geq 2 \mathrm{mg} / \mathrm{dL}$. "Metastatic infection" was defined as a distant site of infection isolated with the same pathogen (K. pneumoniae) as in a pyogenic liver abscess. "Multiple liver abscesses" were defined as $\geq 3$ abscesses detected by imaging. The Acute Physiology and Chronic Health Evaluation II (APACHE II) score was estimated within $48 \mathrm{~h}$ after hospital admission.

\section{Microbiology laboratory procedures}

The VITEK 2 system (bioMérieux, Marcy l'Etoile, France) or matrix-assisted laser desorption-ionization time-offlight mass spectrometry (bioMérieux SA, Marcy l'Etoile, France) was used to confirm bacterial identifications among the available isolates. To determine the capsular types of $K$. pneumoniae, we undertook cps genotyping by the polymerase chain reaction (PCR) detection of K serotype-specific alleles at $w z y$ and $w z x$ loci, including $\mathrm{K} 1$, $\mathrm{K} 2$, K5, K20, K54, and K57, as described previously [16]. These capsular types are thought to be the most closely associated with community invasive disease or pathogenicity [24].

\section{Statistical analyses}

Data were analyzed using SPSS ver. 17 (SPSS, Chicago, IL, USA). Chi square or Fisher's exact tests were carried out for categorical data. The two-tailed Student's $t$ test or Mann-Whitney $U$ test was done for numerical data. A $P$ value $<0.05$ was considered statistically significant.

\section{Results}

\section{Clinical characteristics of KPLA in patients with or without DM}

A total of 255 patients diagnosed with KPLA during the study period were enrolled. 25 patients were excluded: 6 patients were infected by multidrug-resistant $K$. pneumoniae nosocomially, 6 patients developed a liver abscess after trans-hepatic arterial chemoembolization for hepatocellular carcinoma, 1 patient developed a liver abscess after replacing a stent over common bile duct, and 12 patients had polymicrobial liver abscess. Of the remaining 230 patients (149 males), the mean age was $63.8 \pm 15.5$ years. The overall in-hospital mortality of KPLA was $3.5 \%$ (8 patients). More than half the patients $(\mathrm{n}=120,52.2 \%)$ in this study period did not have DM. The rate of DM in patients with KPLA was $44.4 \%$ in $2011,57.9 \%$ in $2012,44.9 \%$ in 2013 , as low as $35.0 \%$ in 2014, and $53.5 \%$ in 2015. 
To further characterize non-diabetic patients with KPLA, Table 1 details the comparisons between diabetic and non-diabetic patients with KPLA. More male patients were in diabetic than non-diabetic group (72.7 vs $57.5 \%, P=0.016)$. Diabetic patients had a significantly higher Charlson comorbidity index $(2.4 \pm 1.9$ vs $1.1 \pm 1.8, P<0.001)$ than those without diabetes. The prevalence of gas-forming abscess (16.4 vs $1.7 \%$, $P=<0.001)$ was significantly higher in diabetic than non-diabetic patients. The APACHE II score was significantly higher in diabetic patients $(14.5 \pm 7.4$ vs

Table 1 Clinical characteristics of diabetic and non-diabetic patients with KPLA

\begin{tabular}{|c|c|c|c|}
\hline & $\begin{array}{l}\text { Non-DM } \\
(n=120)\end{array}$ & $\begin{array}{l}\text { DM } \\
(n=110)\end{array}$ & $p$ \\
\hline Age in years & $64.1 \pm 16.3$ & $63.5 \pm 14.6$ & 0.779 \\
\hline Male & $69(57.5)$ & $80(72.7)$ & 0.016 \\
\hline Charlson score & $1.1 \pm 1.8$ & $2.4 \pm 1.9$ & $<0.001$ \\
\hline \multicolumn{4}{|l|}{ Underlying disease } \\
\hline Malignancy & $11(9.2)$ & $10(9.1)$ & 0.984 \\
\hline Alcoholism & $4(3.3)$ & $8(7.3)$ & 0.180 \\
\hline Chronic kidney disease & $4(3.3)$ & $8(7.3)$ & 0.180 \\
\hline Liver cirrhosis & $5(4.2)$ & $1(0.9)$ & 0.215 \\
\hline Congestive heart failure & $8(6.7)$ & $5(4.5)$ & 0.487 \\
\hline Chronic lung disease & $5(4.2)$ & $5(4.5)$ & 1.000 \\
\hline Cerebrovascular accident & $11(9.2)$ & $8(7.3)$ & 0.602 \\
\hline APACHE II score & $12.3 \pm 6.9$ & $14.5 \pm 7.4$ & 0.018 \\
\hline $\mathrm{HbA} 1 \mathrm{c}$ & $5.6 \pm 1.2$ & $9.2 \pm 2.6$ & $<0.001$ \\
\hline Capsular type $\mathrm{K} 1$ and $\mathrm{K} 2$ & $94(81.7)^{\mathrm{a}}$ & $70(70.7)^{\mathrm{b}}$ & 0.057 \\
\hline Wild-type antibiotic susceptibility & $113(94.2)$ & $103(93.6)$ & 0.867 \\
\hline Origin & & & 0.749 \\
\hline Cryptogenic & $103(85.8)$ & $96(87.3)$ & \\
\hline Biliary tract origin & $17(14.2)$ & $14(12.7)$ & \\
\hline Abscess location & & & 0.408 \\
\hline Right lobe & $77(64.2)$ & $79(71.8)$ & \\
\hline Left lobe & $21(17.5)$ & $17(15.5)$ & \\
\hline Both lobes & $22(18.3)$ & $14(12.7)$ & \\
\hline Abscess size & & & 0.419 \\
\hline$<5 \mathrm{~cm}$ & $51(42.5)$ & $41(37.3)$ & \\
\hline$\geq 5 \mathrm{~cm}$ & $69(57.5)$ & $69(62.7)$ & \\
\hline Gas-forming abscess & $2(1.7)$ & $18(16.4)$ & $<0.001$ \\
\hline Multiple abscesses & $18(15.0)$ & 15 (13.6) & 0.768 \\
\hline Metastatic infection & $6(5.0)$ & $12(10.9)$ & 0.096 \\
\hline \multicolumn{4}{|l|}{ Outcomes } \\
\hline ICU admission & $23(19.2)$ & $28(25.5)$ & 0.252 \\
\hline Hospital days & $26.9 \pm 16.5$ & $32.3 \pm 38.5$ & 0.178 \\
\hline Mortality & $4(3.4)$ & $4(3.6)$ & 0.910 \\
\hline
\end{tabular}

Data are presented as mean \pm SD or frequency with percentage (\%)

a 115 isolates were available for genotyping

b 99 isolates were available for genotyping
Table 2 Clinical characteristics of patients with KPLA stratified by non-diabetic and diabetic with optimal glycemic control

\begin{tabular}{|c|c|c|c|}
\hline & $\begin{array}{l}\text { Non-DM } \\
(n=120)\end{array}$ & $\begin{array}{l}\text { DM with } \\
\text { HbA1c }<7 \% \\
(n=19)\end{array}$ & $p$ \\
\hline Age in years & $64.1 \pm 16.3$ & $69.5 \pm 12.9$ & 0.166 \\
\hline Male & $69(57.5)$ & $13(68.4)$ & 0.369 \\
\hline Charlson score & $1.1 \pm 1.8$ & $2.0 \pm 1.4$ & 0.047 \\
\hline \multicolumn{4}{|l|}{ Underlying disease } \\
\hline Malignancy & $11(9.2)$ & $3(15.8)$ & 0.408 \\
\hline Alcoholism & $4(3.3)$ & $0(0)$ & 1.000 \\
\hline Chronic kidney disease & $4(3.3)$ & $3(15.8)$ & 0.054 \\
\hline Liver cirrhosis & $5(4.2)$ & $0(0)$ & 1.000 \\
\hline Congestive heart failure & $8(6.7)$ & $2(10.5)$ & 0.627 \\
\hline Chronic lung disease & $5(4.2)$ & $1(5.3)$ & 0.593 \\
\hline Cerebrovascular accident & $11(9.2)$ & $0(0)$ & 0.361 \\
\hline APACHE II score & $12.3 \pm 6.9$ & $14.9 \pm 8.4$ & 0.136 \\
\hline Capsular type K1 and K2 & $94(81.7)^{\mathrm{a}}$ & $12(75.0)^{b}$ & 0.506 \\
\hline $\begin{array}{l}\text { Wild-type antibiotic } \\
\text { susceptibility }\end{array}$ & $113(94.2)$ & $17(89.5)$ & 0.355 \\
\hline Origin & & & 1.000 \\
\hline Cryptogenic & $103(85.8)$ & $17(89.5)$ & \\
\hline Biliary tract origin & $17(14.2)$ & $2(10.5)$ & \\
\hline Abscess location & & & 0.937 \\
\hline Right lobe & $77(64.2)$ & $12(63.2)$ & \\
\hline Left lobe & $21(17.5)$ & $4(21.1)$ & \\
\hline Both lobes & $22(18.3)$ & $3(15.8)$ & \\
\hline Abscess size & & & 0.369 \\
\hline$<5 \mathrm{~cm}$ & $51(42.5)$ & $6(31.6)$ & \\
\hline$\geq 5 \mathrm{~cm}$ & $69(57.5)$ & $13(68.4)$ & \\
\hline Gas-forming abscess & $2(1.7)$ & $3(15.8)$ & 0.018 \\
\hline Multiple abscesses & $18(15.0)$ & $3(15.8)$ & 1.000 \\
\hline Metastatic infection & $6(5.0)$ & $1(5.3)$ & 1.000 \\
\hline \multicolumn{4}{|l|}{ Outcomes } \\
\hline ICU admission & $23(19.2)$ & $3(15.8)$ & 1.000 \\
\hline Hospital days & $26.9 \pm 16.5$ & $28.1 \pm 21.1$ & 0.786 \\
\hline Mortality & $4(3.4)$ & $0(0)$ & 1.000 \\
\hline
\end{tabular}

$12.3 \pm 6.9, P=0.018$ ), whereas rate of ICU admission, length of hospital stay, and in-hospital mortality did not differ between the two groups. Interestingly, capsular types $\mathrm{K} 1$ and $\mathrm{K} 2$ tended to be more prevalent in nondiabetic than diabetic patients ( 81.7 vs $70.7 \%, P=0.057$ ).

\section{Clinical characteristics of KPLA in non-diabetic patients and diabetic patients with optimal glycemic level} We compared the clinical characteristics between nondiabetic patients and diabetic patients with optimal 
Table 3 Distribution of capsular types of $K$. pneumoniae isolates in non-diabetic and diabetic patients with different $\mathrm{HbA1c}$ levels

\begin{tabular}{|c|c|c|c|c|c|}
\hline \multirow[t]{2}{*}{ Genotype } & \multirow{2}{*}{$\begin{array}{l}\text { Non-DM } \\
(n=115)\end{array}$} & \multicolumn{2}{|l|}{ DM } & \multirow[t]{2}{*}{$p^{a}$} & \multirow[t]{2}{*}{$p^{b}$} \\
\hline & & $\begin{array}{l}\mathrm{HbA} 1 \mathrm{c}<7 \% \\
(n=16)\end{array}$ & $\begin{array}{l}\mathrm{HbA} 1 \mathrm{c} \geq 7 \% \\
(n=83)\end{array}$ & & \\
\hline K1 & $66(57.4)$ & $12(75.0)$ & $43(51.8)$ & 0.787 & 0.266 \\
\hline K2 & $28(24.3)$ & $0(0)$ & $15(18.1)$ & 0.094 & 0.557 \\
\hline K5 & $6(5.2)$ & $0(0)$ & $3(3.6)$ & 0.510 & 1.000 \\
\hline K20 & $1(0.9)$ & $1(6.3)$ & $4(4.8)$ & 0.098 & 0.210 \\
\hline K54 & $4(3.5)$ & $1(6.3)$ & $4(4.8)$ & 0.736 & 0.737 \\
\hline K57 & $3(2.6)$ & $1(6.3)$ & $1(1.2)$ & 1.000 & 0.651 \\
\hline $\begin{array}{l}\text { The above } \\
6 \text { capsular } \\
\text { types }\end{array}$ & 108 (93.9) & 15 (93.8) & 70 (84.3) & 0.048 & 0.022 \\
\hline Others & $7(6.1)$ & $1(6.3)$ & $13(15.7)$ & 0.048 & 0.022 \\
\hline
\end{tabular}

Data are presented as frequency with percentage (\%)

a Comparison between Non-DM and DM

b Comparison between group with optimal glycemic level (Non-DM + DM with $\mathrm{HbA} 1 \mathrm{c}<7 \%$ ) and diabetic patients without optimal glycemic level (DM with $\mathrm{HbA} 1 \mathrm{c}$ level $\geq 7 \%$ )

glycemic level (HbA1c <7 \%) (Table 2). Diabetic patients with optimal glycemic level had significantly higher Charlson comorbidity index than those without diabetes $(2.0 \pm 1.4$ vs $1.1 \pm 1.8, \mathrm{P}=0.047)$. Interestingly, gasforming abscesses (15.8 vs $1.7 \%, P=0.018)$ occurred more frequently even in diabetic patients with optimal glycemic level than those without diabetes.

\section{Distribution of K. pneumoniae capsular genotypes in patients with different glycemic levels}

Among 230 patients with KPLA, 214 isolates were available for capsular genotyping. Table 3 shows the distribution of capsular types in patients with different glycemic levels. Overall, the six virulent capsular types (K1, K2, $\mathrm{K} 5$, K20, K54, and K57) accounted for $90.2 \%(\mathrm{n}=193)$ of all K. pneumoniae isolates. Capsular type K1 $(\mathrm{n}=121$, $56.5 \%)$ and $\mathrm{K} 2(\mathrm{n}=43,20.1 \%)$ were the most prevalent strains. We also found that $54.5 \%(66 / 121) \mathrm{K} 1$ isolates and $65.1 \%(28 / 43) \mathrm{K} 2$ isolates were isolated from nondiabetic patients, respectively. Capsular type K1 and K2 isolates had the trend to be more prevalent in non-diabetic than diabetic patients (81.7 vs $70.7 \%, P=0.057$ ). The six virulent capsular types (K1, K2, K5, K20, K54, and K57) were significantly more prevalent in non-diabetic than diabetic patients ( 93.9 vs $85.9 \%, P=0.048$ ).

To further explore the association of glycemic status with the distribution of capsular types, non-diabetic and diabetic patients with $\mathrm{HbA1c}$ level $<7 \%$ were defined as the group with optimal glycemic level. Diabetic patients with $\mathrm{HbA1c}$ level $\geq 7 \%$ were defined as the non-optimal glycemic level group. We found that the six virulent capsular types (K1, K2, K5, K20, K54, and K57) were significantly more prevalent in the group with optimal glycemic level than the group without optimal glycemic level (93.9 vs $84.3 \%, P=0.022$ ).

\section{Discussion}

The present study demonstrated the emergence of KPLA in non-diabetic patients as well as the distribution of capsular types of $K$. pneumoniae in Taiwan. In our 5-year cohort study, more than half of the patients did not have underlying DM. While the rate of gas-forming abscess was significantly higher in diabetic than non-diabetic patients, the clinical outcomes did not differ. Capsular types $\mathrm{K} 1, \mathrm{~K} 2, \mathrm{~K} 5, \mathrm{~K} 20, \mathrm{~K} 54$, and $\mathrm{K} 57$ accounted for the majority of all the K. pneumoniae isolates. These six virulent capsular types were significantly more prevalent in the group with optimal glycemic level than that with non-optimal glycemic level.

In one study conducted from July 1991 to June 1998 in two medical centers (including our hospital) in Taiwan, DM was present in $78.4 \%(105 / 134)$ of patients with KPLA [12]. In one recent review article, $63 \%(323 / 512)$ of patients with KPLA had underlying DM in Taiwan [22]. However, in our present study, DM accounted for only $47.8 \%(110 / 230)$ of KPLA patients in the previous 5 -year period (2011-2015). These findings further imply that DM is not a prerequisite for the development of KPLA anymore. As an endemic disease in Taiwan, physicians should be vigilant about the potential of acquiring KPLA in patients without underlying DM. Despite the emergence of KPLA in non-diabetic patients, we still found a higher rate of gas-forming abscess in diabetic patients. This finding corresponded to our former study that indicated the important role of glycemic level in clinical characteristics of KPLA [11].

The comparison of clinical characteristics between non-diabetic patients and diabetic patients with optimal glycemic level (HbA1c $<7 \%$ ) has never been addressed. We found that gas-forming abscess was significantly more prevalent in diabetic patients with optimal glycemic levels than those without diabetes. But the clinical outcome were not different between these two groups. These results might suggest that previous glycemic exposure still had adverse effects upon immunity against subsequent bacterial infection.

The literature has demonstrated that $\mathrm{K} 1$ and $\mathrm{K} 2$ were the major capsular types causing KPLA [22]. Fang et al. has demonstrated that six capsular types (K1, K2, K5, K20, K54, and K57) accounted for $92 \%$ of the K. pneumoniae isolates during 1997-2005 in a medical center in Taiwan [16]. Similarly, our study disclosed that the six capsular types accounted for $93.9 \%$ of K. pneumoniae 
isolates. However, reports regarding the impact of glycemic status on the distribution of capsular types of $K$. pneumoniae isolates are limited. In the current study, K1 isolates predominated in non-DM group and DM groups with different $\mathrm{HbA1c}$ levels. This result again documented that capsular type $\mathrm{K} 1$ is the major virulent factor causing KPLA. Notably, we found that $65.1 \%$ (28/43) of all K2 isolates occurred in non-diabetic patients, which was higher than the previous report $(4 / 10,40 \%)$ by $\mathrm{Yu}$ et al. [25]. These findings imply that $K$. pneumoniae capsular type K2 is sufficiently virulent to cause severely invasive infection, even in a healthy host. Whether or not the prevalent K2 strains account for the increasing proportion of KPLA in non-diabetic patients remained uncertain. Further studies to explore this issue are needed.

We also found that six virulent capsular types were more prevalent in non-DM than DM group. In terms of the association of glycemic status with the distribution of capsular types, the group with optimal glycemic level (Non-DM and DM with HbA1c level $<7 \%$ ) was infected with these six virulent capsular types more frequently than the DM group with HbA1c level $\geq 7 \%$. It implies that non-DM or healthy persons with KPLA are infected primarily with virulent $K$. pneumoniae capsular types, whereas other capsular types with a relatively lower virulence can still cause liver abscesses, mostly in DM patients without optimal glycemic control [25].

Our study has some limitations. First, this study was conducted in a tertiary medical center in northern Taiwan, and all data were collected from medical records retrospectively. Further studies should be carried out prospectively in other regions or countries to confirm our findings. Second, not all K. pneumoniae isolates were determined for capsular genotyping. Finally, the number of diabetic patients with optimal glycemic level was relatively small in comparison to other groups. Despite these limitations, our study was the first to identify the distribution of K. pneumoniae capsular types in patients with KPLA according to the different glycemic status.

\section{Conclusions}

In conclusion, DM accounted for less than $50 \%$ of KPLA patients in the 5-year cohort in Taiwan. Gas-forming abscesses from KPLA occurred more frequently in diabetic patients. The prevalent virulent capsular types of K. pneumoniae in patients with optimal glycemic level play an important role in the emergence of KPLA in nondiabetic patients. Vaccination for prevention of KPLA is not available currently in Taiwan, and it is expected that the virulent $K$. pneumoniae isolates will continuously occur in hosts without traditional risk factors. Further study with emphasis on the characteristics of KPLA in non-diabetic patients is warranted. The knowledge of distribution of capsular types among patients with different glycemic levels will be crucial for the disease control and prevention in the future.

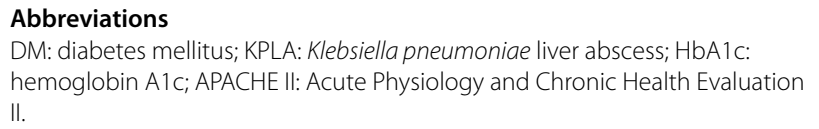

\section{Authors' contributions}

YTL contributed to study concept and design, data interpretation, and manuscript drafting; CC and WCF contributed to data acquisition and analysis; CC contributed to manuscript drafting and data interpretation; and YTL and FDW contributed to the supervision of this investigation. All authors read and approved the final manuscript.

\section{Author details \\ ${ }^{1}$ Department of Medicine, Taipei Veterans General Hospital, Taipei, Taiwan. 2 Division of Infectious Diseases, Department of Medicine, Taipei Veterans General Hospital, Number 201, Section 2, Shih-Pai Road, Beitou District, Taipei 11217, Taiwan. ${ }^{3}$ School of Medicine, National Yang-Ming University, Taipei, Taiwan. ${ }^{4}$ Institute of Emergency and Critical Care Medicine, National Yang-Ming University, Taipei, Taiwan.}

\section{Acknowledgements}

We thank the Medical Science and Technology Building of Taipei Veterans General Hospital for providing experimental space and facilities. Some result from this study was presented as a paper poster at the ASM's Interscience Conference of Antimicrobial Agents and Chemotherapy (ICAAC 2015) in Sandiego, California, United States.

\section{Competing interests}

The authors declare that they have no competing interests.

\section{Availability of data and materials}

The datasets during and/or analyzed during the current study available from the corresponding author on reasonable request. Identifying patient data will not be shared.

\section{Ethics approval and consent to participate}

The study was approved by the Institution Review Board of Taipei Veterans General Hospital. The written informed consent form was waived.

\section{Funding}

This work was supported by grants from the Ministry of Science and Technology in Taiwan (MOST 103-2314-B-075 -078 -MY2), Taipei Veterans General Hospital (V104B-001, and V105B-001), and Szu-Yuan Research Foundation of Internal Medicine (105001).

Received: 28 July 2016 Accepted: 3 October 2016

Published online: 18 October 2016

\section{References}

1. Podschun R, Ullmann U. Klebsiella spp. as nosocomial pathogens: epidemiology, taxonomy, typing methods, and pathogenicity factors. Clin Microbiol Rev. 1998;11:589-603.

2. Meatherall BL, Gregson D, Ross T, Pitout JD, Laupland KB. Incidence, risk factors, and outcomes of Klebsiella pneumoniae bacteremia. Am J Med. 2009; $122: 866-73$

3. Wu HS, Wang FD, Tseng CP, Wu TH, Lin YT, Fung CP. Characteristics of healthcare-associated and community-acquired Klebsiella pneumoniae bacteremia in Taiwan. J Infect. 2012;64:162-8.

4. Lin YT, Jeng YY, Chen TL, Fung CP. Bacteremic community-acquired pneumonia due to Klebsiella pneumoniae: clinical and microbiological characteristics in Taiwan, 2001-2008. BMC Infect Dis. 2010;10:307. 
5. Lin YT, Chen TL, Siu L, Hsu SF, Fung CP. Clinical and microbiological characteristics of community-acquired thoracic empyema or complicated parapneumonic effusion caused by Klebsiella pneumoniae in Taiwan. Eur 」 Clin Microbiol Infect Dis. 2010;29:1003-10.

6. Lin YT, Wang YP, Wang FD, Fung CP. Community-onset Klebsiella pneumoniae pneumonia in Taiwan: clinical features of the disease and associated microbiological characteristics of isolates from pneumonia and nasopharynx. Front Microbiol. 2015;9:122.

7. Chung DR, Lee SS, Lee HR, et al. Emerging invasive liver abscess caused by K1 serotype Klebsiella pneumoniae in Korea. J Infect. 2007;54:578-83.

8. Chan DS, Archuleta S, Llorin RM, Lye DC, Fisher D. Standardized outpatient management of Klebsiella pneumoniae liver abscesses. Int J Infect Dis. 2013;17:e185-8.

9. Wu PF, Chang YY, Lin YT, Wang FD, Chan YJ, Fung CP. Clinical characteristics and economic consequence of Klebsiella pneumoniae liver abscess in Taiwan. J Microbiol Immunol Infect. 2015;48(2):190-7.

10. Lin YT, Liu CJ, Yeh YC, Chen TJ, Fung CP. Ampicillin and amoxicillin use and the risk of Klebsiella pneumoniae liver abscess in Taiwan. J Infect Dis. 2013;208:211-7.

11. Lin YT, Wang FD, Wu PF, Fung CP. Klebsiella pneumoniae liver abscess in diabetic patients: association of glycemic control with the clinical characteristics. BMC Infect Dis. 2013;13:56.

12. Fung $\mathrm{CP}$, Chang FY, Lee SC, et al. A global emerging disease of Klebsiella pneumoniae liver abscess: is serotype $\mathrm{K} 1$ an important factor for complicated endophthalmitis? Gut. 2002;50:420-4.

13. Fang $C T$, Chuang YP, Shun CT, Chang SC, Wang JT. A novel virulence gene in Klebsiella pneumoniae strains causing primary liver abscess and septic metastatic complications. J Exp Med. 2004;199:697-705.

14. Tsai FC, Huang YT, Chang LY, Wang JT. Pyogenic liver abscess as endemic disease, Taiwan. Emerg Infect Dis. 2008;14:1592-600.

15. Wang YP, Liu CJ, Chen TJ, Lin YT, Fung CP. Proton pump inhibitor use significantly increases the risk of cryptogenic liver abscess: a populationbased study. Aliment Pharmacol Ther. 2015;41:1175-81.
16. Fang CT, Lai SY, Yi WC, Hsueh PR, Liu KL, Chang SC. Klebsiella pneumoniae genotype K1: an emerging pathogen that causes septic ocular or central nervous system complications from pyogenic liver abscess. Clin Infect Dis. 2007:45:284-93.

17. Lee SS, Chen YS, Tsai HC, et al. Predictors of septic metastatic infection and mortality among patients with Klebsiella pneumoniae liver abscess. Clin Infect Dis. 2008:47:642-50.

18. Lin YT, Liu CJ, Chen TJ, Fung CP. Long-term mortality of patients with septic ocular or central nervous system complications from pyogenic liver abscess: a population-based study. PLoS ONE. 2012;7:e33978.

19. Lin YT, Liu CJ, Chen TJ, et al. Pyogenic liver abscess as the initial manifestation of underlying hepatocellular carcinoma. Am J Med. 2011;124:1158-64.

20. Wang JH, Liu YC, Lee SS, et al. Primary liver abscess due to Klebsiella pneumoniae in Taiwan. Clin Infect Dis. 1998;26:1434-8.

21. Yang CS, Tsai HY, Sung CS, Lin KH, Lee FL, Hsu WM. Endogenous Klebsiella endophthalmitis associated with pyogenic liver abscess. Ophthalmology. 2007:114:876-80

22. Siu LK, Yeh KM, Lin JC, Fung CP, Chang FY. Klebsiella pneumoniae liver abscess: a new invasive syndrome. Lancet Infect Dis. 2012;12:881-7.

23. Diagnosis and classification of diabetes mellitus. Diabetes Care. 2012;35(Suppl 1):S64-71.

24. Turton JF, Perry C, Elgohari S, Hampton CV. PCR characterization and typing of Klebsiella pneumoniae using capsular type-specific, variable number tandem repeat and virulence gene targets. J Med Microbiol. 2010;59(Pt 5):541-7.

25. Yu WL, Chan KS, Ko WC, Lee CC, Chuang YC. Lower prevalence of diabetes mellitus in patients with Klebsiella pneumoniae primary liver abscess caused by isolates of $\mathrm{K} 1 / \mathrm{K} 2$ than with non-K1/K2 capsular serotypes. Clin Infect Dis. 2007;45:1529-30

\section{Submit your next manuscript to BioMed Central and we will help you at every step:}

- We accept pre-submission inquiries

- Our selector tool helps you to find the most relevant journal

- We provide round the clock customer support

- Convenient online submission

- Thorough peer review

- Inclusion in PubMed and all major indexing services

- Maximum visibility for your research

Submit your manuscript at www.biomedcentral.com/submit
(O) BioMed Central 